Massimo Malacarne, Paolo Formaggioni, Piero Franceschi, Andrea Summer

\title{
Influenza dei fattori genetici sulla resa in formaggio Parmigiano Reggiano e Grana Padano: una minireview
}

\author{
Influence of genetic factors on cheese yield in Parmigiano Reggiano and Grana Padano: \\ a minireview
}

Dipartimento di Scienze Medico-Veterinarie, Università degli Studi di Parma Via del Taglio 10, I-43126, Parma

*Corresponding author:

Paolo Formaggioni

Dipartimento di Scienze Medico-Veterinarie, Università degli Studi di Parma, Via del Taglio

10, I-43126, Parma. Tel. 0521-032614 Email: paolo.formaggioni@unipr.it
Ricevuto il 19 febbraio 2020

Accettato il 15 aprile 2020

DOI: 10.36138/STLC.02.2020.02

\section{Riassunto}

La rassegna si propone di valutare l'influenza dei fattori genetici (polimorfismo genetico delle proteine del latte e razza delle bovine) sulla resa in formaggio Parmigiano Reggiano e Grana Padano, recensendo tutti i lavori che in letteratura si sono occupati di questa tematica. Dopo un breve inquadramento generale sull'effetto dei tipi genetici delle proteine sulla composizione e sull'attitudine del latte alla coagulazione presamica, e di conseguenza sulla resa, è stato valutato, per quanto riguarda la resa in formaggio Parmigiano Reggiano, il confronto tra latti caratterizzati dalle varianti A e B della k-caseina, soprattutto relativamente all'effetto sulla resa della diversa attitudine alla coagulazione presamica; il confronto tra latti caratterizzati dalle varianti $\mathrm{A}$ e $B$ della $\beta$-lattoglobulina; il confronto tra latti di vacche di razza Bruna Italiana e Frisona Italiana. Per quanto riguarda la resa in formaggio Grana Padano è stata valutato il confronto tra latti di vacche di razza Bruna Italiana e Frisona Italiana nel Trentin Grana.

\section{Parole chiave:}
Resa in formaggio
- Parmigiano Reggiano
- Grana Padano
Varianti genetiche di razza
varianti genetiche delle proteine del latte

\section{Abstract}

The review aims to investigate the influence of genetic factors (milk protein polymorphism and cattle breed) on Parmigiano Reggiano and Grana Padano cheese yield, reviewing all the papers that in the literature have dealt with this issue. After a brief general overview on the effect of the genetic types of milk proteins on the composition and on the aptitude of milk to rennet coagulation, and consequently on cheese yield, the following aspects are taken into consideration: concerning the Parmigiano Reggiano cheese yield, the effect of $k$-casein $A$ and $B$ variants, especially concerning the influence of the different rennet-coagulation aptitude on cheese yield; the ef- fect of $\beta$-lactoglobulin $A$ and $B$ variants; the comparison between milks from Italian Brown and Italian Friesian cows. As regards to Grana Padano cheese yield, the comparison between milks from Italian Brown and Italian Friesian cows in Trentin Grana was evaluated.
Keywords:
Cheese yield
- Parmigiano Reggiano
- Grana Padano
- Genetic polymorphism of milk protein
Genetic polymorphism in cattle breeds 


\section{INTRODUZIONE}

I fattori genetici svolgono un ruolo importante nella definizione della qualità del latte per la produzione casearia [1-5]. Le differenze nella produzione di latte e nella composizione chimica sono abbastanza evidenti tra le razze bovine [6-14]. Queste variazioni influenzano le proprietà tecnologiche, ad esempio la resa in formaggio e la sua qualità [10, 15-17].

L'importanza dei fattori genetici sulla resa è stata investigata da numerosi autori; si tratta probabilmente del fattore di variabilità della resa maggiormente investigato in letteratura. A questo proposito, per una più ampia trattazione che non riguardi solo la resa in Parmigiano Reggiano e Grana Padano, si rimanda alle reviews di Buchberger e Dovč [18], Marchini et al. [17] e Bittante et al. [5].

\section{TIPI GENETICI DELLE PROTEINE, COMPOSIZIONE E ATTITUDINE DEL LATTE ALLA COAGULAZIONE PRESAMICA - IL QUADRO GENERALE}

Il polimorfismo delle caseine, e più in generale delle proteine di sintesi mammaria, costituisce un aspetto del tutto particolare del complesso quadro della qualità del latte [18-21].

Le varianti genetiche delle caseine esercitano un'influenza non secondaria nei riguardi delle caratteristiche di coagulazione [5, 18, 22-28]. Gli effetti del polimorfismo delle caseine possono interessare anche alcune variazioni quantitative a livello della produzione di singole proteine specifiche del latte. Infatti, i tipi genetici delle proteine del latte sono in grado di influenzare la produzione quali-quantitativa di caseina e di proteina del siero condizionando, in tal modo, anche il contenuto di proteina del latte $[4,17,18$, 29, 30].

II tipo genetico della k-caseina, ad esempio, esercita una marcata influenza sulle proprietà del latte per la caseificazione. Le due varianti genetiche della $\mathrm{k}$-caseina più ampiamente diffuse sono designate come A e B [31-33]. Rispetto alla k-caseina A, la k-caseina $B$ è associata $a$ un più alto contenuto di caseina nel latte $[26,34]$ e una percentuale più alta di k-caseina sulla caseina totale [30]. Queste caratteristiche comportano micelle più piccole [21, 35] che, a loro volta, portano a migliori proprietà del coagulo. Infatti, il latte contenente la k-caseina B manifesta una maggiore reattività con il caglio ed, in particolare, una migliore attitudine alla formazione del coagulo (in termini di proprietà di coagulazione misurate mediante lattodinamografia) [26], il cui reticolo proteico tende ad essere anche più elastico rispetto a quello che contraddistingue il latte di tipo k-caseina $A$ [36].
Inoltre, la k-caseina BB è anche correlata a una maggiore resa del formaggio; questo risulta evidente in molti tipi di caseificazioni, anche molto diverse tra loro, come il Cheddar [15, 35], il Parmigiano Reggiano [37], la mozzarella a bassa umidità (umidità $<52 \%$; mozzarella per pizza) prodotta con latte parzialmente scremato [38] e la mozzarella propriamente detta, ad elevata umidità (umidità $>52 \%$ ma comunque $\leq 60 \%$ ) [39].

Anche il latte $\beta$-caseina $B$, a sua volta, tende a coagulare in tempi sensibilmente inferiori rispetto a quello di tipo $A$, con effetti importanti sulla velocità di formazione del coagulo e di riflesso anche sulla sua consistenza $[23,24]$.

Sotto questo profilo molto marcata è l'influenza dei tipi genetici della più importante proteina del siero, la $\beta$-lattoglobulina. Le vacche che si distinguono per le varianti $A$ e $B$ di questa proteina producono quantità differenti di $\beta$-lattoglobulina nel latte $[23,24,29$, 40]. Quelle di tipo A, infatti, portano ad un latte più ricco di $\beta$-lattoglobulina e quindi con maggiore quantità di proteina del siero rispetto a quelle di tipo $B$. Le vacche di tipo $B$, quindi, danno un latte mediamente più povero in sieroproteina che, di conseguenza, si presenta più ricco di caseina, con effetti significativi sul rendimento della trasformazione casearia [12, 41].

In seguito riportiamo i principali contributi relativi alla resa in Parmigiano Reggiano e Grana Padano.

\section{CONFRONTO TRA LATTI CARATTERIZZATI DALLE VARIANTI A E B DELLA K-CASEINA NELLA PRODUZIONE DEL FORMAGGIO PARMIGIANO REGGIANO}

Come sottolineano anche Buchberger e Dovč [18], il primo studio sulla relazione tra la resa in formaggio e k-CN AA e k-CN BB, è stato quello di Mariani et al. [37].

In particolare, Mariani et al. [37] hanno valutato l'influenza delle varianti genetiche della k-caseina sul comportamento tecnologico del latte nella produzione del formaggio Parmigiano Reggiano.

Nel periodo giugno-settembre sono state effettuate 12 prove comparative di caseificazione di latte caratterizzato dalle varianti $A \circ B$ della k-caseina, prodotto da vacche di razza Frisona omozigoti per l'uno o per l'altro allele, scelte tra 472 appartenenti a 6 allevamenti diversi. I due gruppi sono stati costituiti abbinando alle 22 vacche disponibili di genotipo k-BB, altrettante vacche di genotipo k-AA, aventi caratteristiche confrontabili per tipo genetico delle altre frazioni proteiche, ordine e stadio di lattazione e produzione di latte. I rilievi effettuati in caseificio e le analisi di laboratorio hanno ampiamente dimostrato che i due tipi di latte presentavano caratteristiche 
differenti: il latte k-BB risultava migliore rispetto al latte k-AA. Infatti i coaguli ottenuti dal latte k-BB erano dotati di maggiore consistenza, la frantumazione delle cagliate risultava meno difficile e i grumi più uniformi; inoltre, le caratteristiche di spurgo, di coesione e, più in generale, le caratteristiche reologiche delle masse caseose prodotte con il latte $\mathrm{k}-\mathrm{BB}$ sono apparse quasi sempre nettamente più favorevoli. Il latte k-BB, rispetto al latte k-AA, si è dimostrato, nel complesso, più idoneo per la lavorazione a formaggio Parmigiano Reggiano. Queste osservazioni sono state confermate in laboratorio mediante lattodinamografia, gelometria e tensiometria.

Il latte k-BB ha dato una resa in formaggio, valutata a 24 ore, pari a 8,04 kg per $100 \mathrm{~kg}$ di latte lavorato, contro i 7,41 kg del latte k$A A$. La differenza di $0,63 \mathrm{~kg}$ a favore del latte $\mathrm{k}$-BB è piuttosto rilevante, poiché essa indica che, con la stessa quantità di latte lavorato, dal latte k-AA si ottenevano $100 \mathrm{~kg}$ di formaggio, mentre dal latte $\mathrm{k}-\mathrm{BB}$ se ne ottenevano $108,5 \mathrm{~kg}$.

Altro dato degno di rilievo è il calo in peso del formaggio nelle prime 24 ore, che è risultato maggiore per il formaggio k-BB. In media, contro un calo del 7,81\% nel formaggio $k-A A$, in quello $k-B B$ si è osservato un calo del $9 \%$. Se si considera che la maggiore perdita di siero avviene nelle prime ore dopo la lavorazione, se ne può dedurre che la massa caseosa ottenuta dal latte k-BB manifesta in questa fase una maggiore capacità di contrazione o di espulsione del siero. Al contrario, nella fase successiva, cioè tra la $24^{a}$ ora $e$ l'uscita dalla salamoia, è il formaggio $k-A A$ che cala maggiormente di peso rispetto al k-BB, ma la differenza è di modesta entità.

\section{PARAMETRI DI COAGULAZIONE E RESA DI LATTI CARATTERIZZATI DALLE VARIANTI A E B DELLA K-CASEINA NELLA PRODUZIONE DEL FORMAGGIO PARMIGIANO REGGIANO}

Losi et al. [42] hanno misurato, mediante lattodinamografia, i parametri di coagulazione di latti caratterizzati dalle due differenti varianti genetiche della k-caseina.

Dall'esame dei risultati ottenuti, è emerso che tali parametri sono profondamente influenzati dalle diverse varianti presenti nel latte. In particolare, per quanto riguarda la produzione di formaggio Parmigiano Reggiano, i parametri di coagulazione misurati risultavano estremamente più favorevoli quando era presente nel latte la variante $B$ della $\mathrm{k}$-caseina, rispetto alla variante $\mathrm{A}$.

Queste osservazioni sono state confermate in caseificio lavorando i latti k-AA e k-BB in caldaie separate.

I risultati ottenuti in questa prova, dimostrano che i latti k-BB sono più adatti per la trasformazione casearia a Parmigiano Reggiano (minore tempo di coagulazione e tempo di rassodamento del coagulo; maggiore consistenza del coagulo), e inoltre si ottengono rese superiori rispetto ai latti k-AA.

\section{CONFRONTO TRA LATTI CARATTERIZZATI DALLE VARIANTI A E B DELLA $\beta$-LATTOGLOBULINA NELLA PRODUZIONE DEL FORMAGGIO PARMIGIANO REGGIANO}

Le varianti genetiche della $\beta$-lattoglobulina hanno una marcata influenza sulla resa in formaggio. Infatti, il latte $\beta$-lattoglobulina B si contraddistingue per una minore percentuale di $\beta$-lattoglobulina, che si traduce in una percentuale più alta di caseine sulla proteina totale, con conseguente aumento della resa in formaggio.

Morini et al. [43] hanno studiato gli effetti delle varianti A e B della $\beta$-lattoglobulina sulla resa in formaggio Parmigiano Reggiano. Nel corso della ricerca sono state prodotte 180 forme di Parmigiano Reggiano, 90 ottenute dal latte di vacche caratterizzate dal genotipo $\beta$-lattoglobulina A e 90 dal latte di vacche $\beta$-lattoglobulina B. I due gruppi risultavano confrontabili tra loro per quanto riguarda il genotipo nei loci $\alpha_{s}-$, e k-caseina, lo stadio ed ordine di lattazione, l'alimentazione e la tecnologia di allevamento.

La ricerca è stata articolata in due periodi sperimentali; in entrambi il latte $\beta$-lattoglobulina $B$ ha fatto registrare il più alto indice $\mathrm{di}$ caseina $\left(78,40 \mathrm{~B}\right.$ vs 76,16 A \%, $1^{\circ}$ periodo; 79,02 B vs 77,00 A \%, $2^{\circ}$ periodo) rispetto al latte $\beta$-lattoglobulina $A$ e le differenze sono risultate statisticamente significative. Per contro il latte $\beta$-lattoglobulina $A$ è risultato più ricco in sieroproteine $(0,52 \mathrm{~A}$ vs 0,47 B g/100g, $1^{\circ}$ periodo; 0,56 A vs 0,48 B g/100g, $2^{\circ}$ periodo), e conseguentemente, nel primo periodo sperimentale, caratterizzato da un minor contenuto in caseine rispetto alla variante $B$, con differenze anche in questo caso significative. Nel secondo periodo sperimentale, invece, a causa del valore molto più elevato della proteina grezza nel caso della variante $\mathrm{A}(3,37 \mathrm{~A}$ vs 3,27 $B ; g / 100 \mathrm{~g}$ ), il contenuto di caseina non ha fatto registrare differenze statisticamente significative fra le due varianti.

Questi risultati sono stati confermati inoltre dai dati relativi ai pesi e alle rese di lavorazione dei formaggi prodotti. Le differenze relative alla quota di azoto caseinico hanno determinato una maggiore resa per ogni $100 \mathrm{~kg}$ di latte lavorato, pari a circa $200 \mathrm{~g}$ di formaggio in più a favore delle vacche $\beta$-lattoglobulina $B$.

Le rese a 24 ore, dopo salatura e dopo 1 anno, di latti omozigoti per le varianti A e B della $\beta$-lattoglobulina, nei due periodi di sperimentazione, sono riportati in Tabella I [43].

In particolare, nel primo periodo, all'uscita della salamoia, le 54 
forme prodotte con il latte $\beta$-lattoglobulina $B$ pesavano mediamente $1 \mathrm{~kg}$ in più di quelle $\beta$-lattoglobulina $A$; tale differenza era statisticamente significativa $(P<0,05)$. Nel secondo periodo sperimentale invece, gli autori non hanno riscontrato differenze di resa statisticamente significative tra i due tipi di latte lavorati. Va ricordato però il fatto che il latte $\beta$-lattoglobulina $B$, nel secondo periodo sperimentale, come già sottolineato, aveva un contenuto di proteina totale più basso rispetto a quello $\beta$-lattoglobulina $A$ (proteina vera: $3,16 \mathrm{~A}$ vs $3,06 \mathrm{~B} ; \mathrm{g} / 100 \mathrm{~g}$ ). Tuttavia per effetto del maggiore indice di caseina nel latte $\beta$-lattoglobulina $B$ (vedi dati già riportati sopra), il contenuto in caseina è risultato praticamente identico nei due tipi di latte (mediamente 2,60 g/100g).

\section{LATTE DI BRUNA E DI FRISONA NELLA CASEIFICAZIONE A PARMIGIANO REGGIANO: EFFETTI SULLA RESA}

Malacarne et al. [44] riportano i risultati di uno studio finalizzato al confronto tra la composizione chimica di base, la principale distribuzione delle frazioni proteiche, le proprietà di coagulazione e la resa del formaggio Parmigiano Reggiano di latte in caldaia proveniente da vacche Brune Italiane (BI) e Frisone Italiane (FI). Nello studio sono stati impiegati caseifici di Parmigiano Reggiano che lavorano separatamente il latte degli allevamenti di Bruna Italiana da quelli di Frisona Italiana. Sono state condotte 13 prove di caseificazione in 10 diversi caseifici commerciali. Lo studio è stato condotto da marzo a ottobre 2003. Per ogni caseificazione in ciascun caseificio, sono stati trasformati in parallelo circa $1100 \mathrm{~kg}$ di latte di vacche $\mathrm{Bl}$ e altrettanti di vacche FI. Gli animali coinvolti nello studio provenivano da allevamenti con comparabili pratiche di gestione, dimensioni, ubicazione, numero di lattazioni e giorni di mungitura. II latte di Bl è risultato caratterizzato da un più alto contenuto di caseina $(27,1 \mathrm{vs} 23,7 \mathrm{~g} / \mathrm{kg} ; \mathrm{P}<0,0001)$ rispetto al latte di Fl. Anche le sieroproteine $(+13,0 \%$; $P<0,0001)$ e NPNx6,38 $(+12,5 \% ; \mathrm{P}<0,01)$ risultavano più alti nel latte $\mathrm{BI}$ rispetto al latte $\mathrm{FI}$. L'indice di caseina non era statisticamente diverso tra le due razze. Come previsto, non sono state osservate differenze relative al contenuto di grasso del latte. II contenuto di lattosio era più alto (+ $20 \%$; $\mathrm{P}<0,05)$ nel latte $\mathrm{BI}$. II tempo di rassodamento del coagulo $\left(\mathrm{k}_{20}\right)$ del latte di $\mathrm{BI}$ era notevolmente inferiore a quello del latte di FI (6,6 vs 10,0 min; $P<0,001)$. Ciò implica un tasso più elevato di aggregazione di micelle di para-caseina per il latte BI. II coagulo del latte BI presentava migliori proprietà reologiche e minori perdite di grasso nel siero. Anche la resa del formaggio Parmigiano Reggiano a 24 ore era notevolmente superiore (+ 12,48\%; $\mathrm{P}<0,0001)$ per il latte $\mathrm{BI},+0,99 \mathrm{~kg}$ di formaggio per ogni $100 \mathrm{~kg}$ di latte in caldaia (Figura 1).
Tabella I. Resa in Parmigiano Reggiano a 24 ore, dopo salatura e dopo 1 anno di latti omozigoti per varianti A e B della $\beta$-lattoglobulina (Morini et al., 1982).

Table I. Parmigiano Reggiano cheese yield at 24 hours, after salting and after 1 year of homozygous milks for variants $A$ and $B$ of $\beta$-lactoglobulin (Morini et al., 1982).

\begin{tabular}{|c|c|c|c|}
\hline \multicolumn{4}{|c|}{$1^{\circ}$ periodo di sperimentazione } \\
\hline & $\beta-\operatorname{Lg} A$ & $\beta-\operatorname{Lg} B$ & \\
\hline Resa a 24 ore & $7,62 \%$ & $7,77 \%$ & $S$ \\
\hline Resa dopo salatura & $7,17 \%$ & $7,35 \%$ & S \\
\hline Resa dopo 1 anno & $6,54 \%$ & $6,71 \%$ & $S$ \\
\hline \multicolumn{4}{|c|}{$1^{\circ}$ periodo di sperimentazione } \\
\hline & $\beta-\operatorname{Lg} A$ & $\beta-\operatorname{Lg} B$ & \\
\hline Resa a 24 ore & $8,34 \%$ & $8,33 \%$ & NS \\
\hline Resa dopo salatura & $7,98 \%$ & $8,03 \%$ & NS \\
\hline Resa dopo 1 anno & $7,34 \%$ & $7,34 \%$ & NS \\
\hline
\end{tabular}

S: differenza significativa; NS: differenza non significativa

Il latte $\mathrm{BI}$ è stato caratterizzato da una maggiore resa in formaggio in tutte le lavorazioni e le differenze fra le due razze erano mediamente di circa $1 \mathrm{~kg}$ di formaggio per $100 \mathrm{~kg}$ di latte in caldaia, con un intervallo che andava da un minimo di 0,44 a un massimo di $1,72 \mathrm{~kg}$ di formaggio per $100 \mathrm{~kg}$ di latte in caldaia.

\section{LATTE DI BRUNA E DI FRISONA NELLA CASEIFICAZIONE A TRENTIN GRANA: EFFETTI SULLA RESA}

De Marchi et al. [45] hanno confrontato il latte di due diverse razze, Brown Swiss (BS) e Holstein Friesian (HF), e i parametri di caseificazione e di resa, nella produzione di 3 diversi formaggi; tra questi, a noi interessa particolarmente la produzione di Trentin Grana, in quanto questo formaggio fa parte della DOP Grana Padano, pur presentando un Disciplinare a sé stante con caratteristiche simili a quello del Parmigiano Reggiano.

Lo scopo di questo studio era di studiare l'effetto di 3 tipi di latte, di vacche di razza Holstein Friesian, di Brown Swiss e misti di entrambe le razze, sulle caratteristiche del latte in caldaia, sulla resa del formaggio e sulla sua qualità in 3 differenti tipiche caseificazioni italiane (Casolet, Vezzena e Trentin Grana). Sono state coinvolte centoqua- 
rantaquattro bovine ( $66 \mathrm{HF}$ e $78 \mathrm{BS}$ ) e sono state valutate un totale di 24 caldaie. Alla fine sono state analizzate 30, 21 e 16 forme di formaggio Casolet, Vezzena e Trentin Grana, rispettivamente. Le vacche BS hanno prodotto il $9 \%$ in meno di latte al giorno rispetto alle vacche HF, ma il latte ha mostrato un maggiore contenuto di proteina, caseina, acidità titolabile e un migliore tempo di coagulazione $\mathrm{e}$ consistenza del coagulo rispetto al latte HF.

II formaggio prodotto con latte Brown Swiss ha avuto una resa maggiore rispetto a quello ottenuto da Holstein Friesian. II latte misto ha mostrato valori, in media, intermedi tra le caratteristiche dei due latti, e questa tendenza è stata confermata nella resa del formaggio a diversi tempi di maturazione. A 24 ore dalla produzione, la resa (\%) del latte BS era notevolmente maggiore rispetto a quella del latte $\mathrm{HF}$, in particolare $+12 \%$ nel caso del Trentin Grana. La resa del formaggio Trentin Grana è stata registrata anche a 180 e 365 giorni di maturazione e, in tutti i casi, BS ha mostrato una resa maggiore di HF. Gli autori affermano che l'aumento della resa del latte BS a 24 ore non sia completamente spiegabile dal maggiore contenuto di proteina+grasso (per Trentin Grana, $+12 \%$ per la resa contro $+9 \%$ di proteina+grasso). II latte BS ha dato una resa extra dal 3 al $9 \%$ rispetto al valore atteso sulla base della composizione del latte.

\section{CONCLUSIONI}

In conclusione, si può affermare, sulla scorta dei lavori prima descritti, che il latte caratterizzato dalla k-caseina BB permette di ottenere una resa in formaggio Parmigiano Reggiano maggiore rispetto a quella del latte caratterizzato dalla k-caseina $A A$; esso, quindi, si dimostra più idoneo per la caseificazione a formaggio Parmigiano Reggiano. Anche il coagulo ottenuto dal latte k-caseina BB si rivela dotato di maggiore consistenza, e le caratteristiche di spurgo, di coesione e, più in generale, le caratteristiche reologiche delle masse caseose prodotte con il latte k-caseina BB sono più favorevoli. Anche le varianti genetiche della $\beta$-lattoglobulina hanno una marcata influenza sulla resa in formaggio Parmigiano Reggiano. Infatti, il latte $\beta$-lattoglobulina B si contraddistingue per una minore percentuale di $\beta$-lattoglobulina nel latte, che si traduce in una percentuale più alta di caseine sulla proteina totale, con conseguente aumento della resa in formaggio. Da questo punto di vista, i risultati ottenuti per la lavorazione a formaggi duri e a lunga maturazione si pone perfettamente in linea con i contributi sperimentali osservati per la produzione di altre tipologie di formaggi, alcuni dei quali sono riportati nell'introduzione del presente lavoro.

\section{Figura 1. Resa a 24 ore in formaggio Parmigiano Reggiano di latte di bovine di razza Frisona Italiana e latte di bovine di razza Bruna Italiana (13 caseificazioni; 10 caseifici diversi) (Malacarne et al., 2006).}

Figure 1. 24-hour Parmigiano Reggiano cheese yield of milk from Italian Frisian cows and milk from Italian Brown cows (13 cheesemakings; 10 different dairies) (Malacarne et al., 2006).

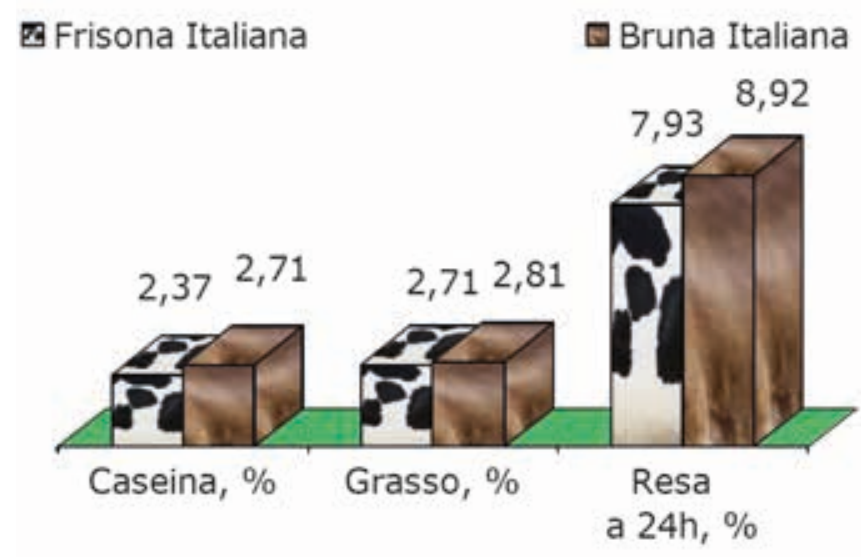

II confronto fra razze nella produzione del Parmigiano Reggiano ha fatto evidenziare differenze importanti per quanto riguarda la resa in formaggio Parmigiano Reggiano e Trentin Grana (quest'ultimo facente parte della DOP Grana Padano, ma simile, per Disciplinare, al Parmigiano Reggiano). Infatti, il latte delle vacche di razza Bruna è risultato caratterizzato da un più alto contenuto di caseina rispetto al latte delle vacche di razza Frisona. Di conseguenza, la resa del formaggio Parmigiano Reggiano è risultata notevolmente superiore per il latte di Bruna rispetto a quello di Frisona. Anche questi risultati appaiono in linea con quanto riscontrato per la produzione di altre tipologie di formaggi.

Bisogna tuttavia tenere conto del fatto che, a fronte di un fiorire di studi sul polimorfismo in generale o nella produzione di altre tipologie di formaggi, gli studi che riguardano gli effetti delle varianti genetiche delle proteine del latte sulla resa in formaggi a pasta dura e a lungo periodo di maturazione, come il Parmigiano Reggiano, sono pochi e piuttosto datati. Questa scarsità di contributi sperimentali può essere in parte dovuta alla difficoltà logistica nella rilevazione delle rese; non tanto per la difficoltà di 
pesare le forme, per le quali esistono apposite bilance, quanto per la difficoltà di avere una misura accurata del latte in caldaia: ovviamente l'approssimativa misura che il casaro ottiene attraverso l'asta graduata non si rivela adeguata per un lavoro scientifico serio. Tale difficoltà può essere superata attraverso un contalitri, che misuri il volume di latte (sia magro che intero del mattino) nel momento in cui esso viene introdotto nella caldaia (e si misuri, contemporaneamente, la densità in caseificio alla temperatura effettiva alla quale si trova il latte), oppure attraverso celle di carico poste sotto a vasconi appositi, prima di rimettere in caldaia il latte. Tutto questo, come si può immaginare, moltiplicato per un numero elevato di osservazioni, non può che risultare particolarmente laborioso e dispendioso in termini di tempo e di risorse economiche, tanto più che molti casari non si rivelano particolarmente entusiasti di queste incursioni nella loro routine quotidiana.

A queste considerazioni, possiamo aggiungere che, rispetto ai lavori riportati, sono state successivamente scoperte numerose altre varianti genetiche, soprattutto per la k-caseina [18]; tuttavia, la maggior parte di queste varianti non è stata ancora studiata per quanto riguarda la capacità di caseificazione, a maggior ragione in formaggi a pasta dura e lunga maturazione, e, quando questo è avvenuto, non è stata misurata la resa in formaggio. Si tratta di un'altra prospettiva molto interessante e un campo di ricerca e applicazione futura, con ripercussioni economiche non indifferenti sulle nuove possibilità di selezione all'interno delle razze bovine.

Come ulteriore considerazione, bisogna tenere conto del fatto che nella maggior parte delle pubblicazioni, anche nella produzione di altri formaggi, l'attenzione si è concentrata sugli effetti dei singoli alleli. Tuttavia, a causa dello stretto legame tra i geni delle caseine, è importante studiare più approfonditamente gli effetti degli aplotipi sulle caratteristiche di coagulazione e sulla resa in formaggio. Al momento sono disponibili solo poche informazioni sulle associazioni tra aplotipi, composizione del latte e indice di caseina; è stata inoltre studiata la relazione tra aplotipi e proprietà di coagulazione del latte e l'associazione tra aplotipi e resa in formaggio [18]. Naturalmente questi studi non riguardano i formaggi duri a lunga maturazione, quali Parmigiano Reggiano e Grana Padano.

Ricordiamo, infine, che sarebbe importante caratterizzare la composizione e le proprietà di coagulazione (soprattutto in riferimento alla resa in formaggio) delle razze autoctone e tradizionali del Parmigiano Reggiano, quali la rossa Reggiana e la Bianca Valpadana o Modenese, che possono costituire un patrimonio prezioso di alleli e possono rivelarsi pregiate per la produzione di formaggi duri a lunga maturazione, per i quali a lungo queste razze sono state votate.

\section{CONFLITTO DI INTERESSE}

Non esistono conflitti di interesse di ordine economico o di altro tipo sull'articolo presentato.

\section{BIBLIOGRAFIA}

1. Martin $P$, Ferranti $P$, Leroux $C$, Addeo F. Non-bovine caseins: quantitative variability and molecular diversity. In Advanced dairy chemistry, Vol. 1, 3rd Edition, Part A, (Eds PF Fox, PLH McSweeney). London: Kluwer Academic, 2003, pp. 277-317.

2. Coulon JB, Delacroix-Buchet A, Martin B, Pirisi A. Relationships between ruminant management and sensory characteristics of cheeses: a review. Lait. 2004;84:221-41

3. Summer A, Malacarne M, Formaggioni P, Franceschi P, Mariani P. Genetic factors and dairy-technological valorisation of the milk. Sci Tecn Latt-Cas. 2009;60(5):415-440.

4. Marchini $C$, Malacarne $M$, Franceschi $P$, Formaggioni $P$, Summer $A$, Mariani P. Genetic factors, casein micelle structural characteristics and rennet coagulation properties of milk. Ann Fac Medic Vet Univ Parma. 2010;30:103-121. 5. Bittante G, Penasa M, Cecchinato A. Invited review: Genetics and modelling of milk coagulation properties. J Dairy Sci. 2012;95(12):6843-6870.

6. Macheboeuf D, Coulon JB, D'Hour P. Effect of breed, protein genetic variants and feeding on cows' milk coagulation properties. J Dairy Res. 1993;60:43-54.
7. Malossini F, Bovolenta S, Piras C, Dalla Rosa M, Ventura W. Effect of diet and breed on milk composition and rennet coagulation properties. Ann Zootech. 1996;45:29-40.

8. Campo P, Licitra G, Gelsomino R, Corallo L, Carpino S, Barbano DM. Composition of milk from Modicana and other breeds of dairy cattle in Sicily. Milchwissenschaft. 1999;54:426-430.

9. Chiofalo V, Maldonato R, Martin B, Dupont D, Coulon JB. Chemical composition and coagulation properties of Modicana and Holstein cows' milk. Ann Zootech. 2000;49:497-503.

10. Auldist M, Mullins C, O'Brien B, O’Kennedy BT, Guinee T. Effect of cow breed on milk coagulation properties. Milchwissenschaft. 2002;57:140-143.

11. Auldist MJ, Johnston KA, White NJ, Fitzsimons WP, Boland MJ. A comparison of the composition, coagulation characteristics and cheesemaking capacity of milk from Friesian and Jersey dairy cows. J Dairy Res. 2004;71:51-57.

12. Wedholm A, Larsen LB, Lindmark-Månsson $H$, Karlsson $A H, A$ ndrén $A$. Effect of protein composition on the cheese-making properties of milk from individual dairy cows. J Dairy Sci. 2006;89(9):3296-3305. 
13. De Marchi M, Dal Zotto R, Cassandro M, Bittante G. Milk coagulation ability of five dairy cattle breed. J Dairy Sci. 2007;90:3986-3992.

14. Gustavsson F, Buitenhuis AJ, Johansson M, Bertelsen HP, Glantz M, Poulsen NA, Lindmark Månsson H, Stålhammar H, Larsen LB, Bendixen C, Paulsson M, Andrén A. Effects of breed and casein genetic variants on protein profile in milk from Swedish Red, Danish Holstein, and Danish Jersey cows. J Dairy Sci. 2014;97(6):3866-3877.

15. Marziali AS, Ng-Kwai-Hang KF. Relationships between milk protein polymorphisms and cheese yielding capacity. J Dairy Sci. 1986;69:1193-1201. 16. Mistry VV, Brouk MJ, Kasperson KM, Martin E. Cheddar cheese from milk of Holstein and Brown Swiss cows. Milchwissenschaft. 2002;57:19-23.

17. Marchini $C$, Malacarne $M$, Franceschi $P$, Formaggioni $P$, Summer A, Mariani P. Milk protein genetic combinations, mineral composition and rennetcoagulation properties of the milk. Ann Fac Medic Vet Univ Parma. 2011;31:45-64.

18. Buchberger J, Dovč P. Lactoprotein genetic variants in cattle and cheese making ability. Food Tech Biotech. 2000;38(2):91-98.

19. Formaggioni P, Summer A, Malacarne M, Mariani P. Milk protein polymorphism: Detection and diffusion of the genetic variants in Bos genus. Ann Fac Medic Vet Univ Parma. 1999;19:127-165.

20. Ng-Kwai-Hang KF, Grosclaude F. Genetic polymorphism of milk protein. In Advanced dairy chemistry, Vol. 1, 3rd Edition, Part B, (Eds PF Fox, PLH McSweeney). London: Kluwer Academic, 2003, pp. 739-816.

21. Hristov P, Neov B, Sbirkova H, Teofanova D, Radoslavov G, Shivachev B. Genetic polymorphism of kappa casein and casein micelle size in the Bulgarian Rhodopean cattle breed. Biotechnol Anim Husb. 2014;30(4):561-570.

22. Mariani P, Pecorari M. Genetic factors, milk cheesemaking aptitude and cheese yield. Sci Tecn Latt-Cas. 1987;38:286-326.

23. Mariani $P$. Fattori genetici, proprietà tecnologico-casearie e resa del latte in formaggio grana. Atti Convegno "La trasformazione del latte in grana: tecnologie e qualità", 1988;9-30.

24. Mariani P, Summer A. Polimorfismo delle proteine ed attitudine tecnologico-casearia del latte. Sci Tecn Latt-Cas. 1999;50 (3):197-230.

25. Di Stasio L, Mariani P. The role of protein polymorphism in the genetic improvement of milk production. Zootec Nutr Anim. 2000;26(3):69-90.

26. Comin A, Cassandro M, Chessa S, Ojala M, Dal Zotto R, De Marchi M, Carnier P, Gallo L, Pagnacco G, Bittante G. Effects of composite $\beta$ - and k-casein genotypes on milk coagulation, quality, and yield traits in Italian Holstein cows. J Dairy Sci. 2008;91:4022-4027.

27. Bugeac $T$, Maciuc V, Creanga S. Genetic parameters of milk yield and quality traits in Holstein-Friesian cows. Bulletin of University of Agricultural Sciences and Veterinary Medicine Cluj-Napoca. Anim Sci Biotechnol. 2013;70(1):150-154

28. Poulsen NA, Bertelsen HP, Jensen HB, Gustavsson F, Glantz M, Månsson HL, Andrén A, Paulsson M, Bendixen C, Buitenhuis AJ, Larsen LB. The occurrence of noncoagulating milk and the association of bovine milk coagulation properties with genetic variants of the caseins in 3 Scandinavian dairy breeds. J Dairy Sci. 2013;96(8):4830-4842.

29. Martin P, Szymanowska M, Zwierzchowski L, Leroux C. The impact of genetic polymorphisms on the protein composition of ruminant milks. Repr Nutr Develop. 2002;42(5):433-459.

30. Heck JML, Schennink A, van Valenberg HJF, Bovenhuis H, Visker MHPW, van Arendonk JAM, van Hooijdonk ACM. Effects of milk protein variants on the protein composition of bovine milk. J Dairy Sci. 2009;92:1192-202.
31. Summer A, Santus E, Casanova L, Joerg H, Rossoni A, Nicoletti C, Donofrio G, Mariani P, Malacarne M. Short communication: characterization of a monoclonal antibody for k-casein B of cow's milk. J Dairy Sci. 2010;93:796-800. 32. Nicoletti C, Rossoni A, Santus E, Summer A. Variability of the content of $\mathrm{k}$-casein $\mathrm{B}$ in individual milk samples. Variabilità del contenuto di k-caseina $B$ in campioni di singole vacche. Sci Tecn Latt-Cas. 2011;62(4):283-287.

33. Rossoni A, Nicoletti C, Santus E, Malacarne M. Analysis of the contents of k-casein B in bulk milk samples. Sci Tecn Latt-Cas. 2011;62(4):267-273.

34. Bugeac T, Balteanu VA, Creangă Ș. Kappa-Casein Genetic Variants and Their Relationships with Milk Production and Quality in Montbéliarde Dairy Cows. Bulletin of University of Agricultural Sciences and Veterinary Medicine Cluj-Napoca. Anim Sci Biotechnol. 2013;70(1):193-194.

35. Walsh CD, Guinee TP, O'Kennedy BT, Reville WD, Harrington D, Murphy $\mathrm{JJ}$, Fitzgerald RJ. The influence of $\mathrm{k}$-casein genetic variant on casein micelle size, rennet gel microstructure and Cheddar cheesemaking properties. Int Dairy J. 1998:8:707-714.

36. Hallén E, Allmere T, Näslund J, Andrén A, Lundén A. Effect of genetic polymorphism of milk proteins on rheology of chymosin-induced milk gels. Int Dairy J. 2007;17:791-799.

37. Mariani P, Losi G, Russo V, Castagnetti GB, Grazia L, Morini D, Fossa E. Prove di caseificazione con latte caratterizzato dalle varianti A e B della k-caseina nella produzione del formaggio Parmigiano-Reggiano. Sci Tecn LattCas. 1976;27:208-27.

38. Walsh CD, Guinee TP, Harrington D, Mehra R, Murphy J, Fitzgerald RJ. Cheesemaking, compositional and functional characteristics of low-moisture part-skim Mozzarella cheese from bovine milks containing k-casein AA, AB or BB genetic variants. J Dairy Res. 1998;65:307-315.

39. Franceschi P, Malacarne M, Faccia M, Rossoni A, Santus E, Formaggioni P, Summer A. New insights of cheese yield capacity between Italian Brown and Italian Friesian milks in the production of high moisture mozzarella. Food Technol Biotechnol. 2020;accepted and in press.

40. Meza-Nieto MA, González-Córdova AF, Becerril-Pérez CM, Ruiz-Lopez FJ, Diaz-Rivera P, Vallejo-Cordoba B. Genetic polymorphism of $\beta$-lactoglobulin in cow's milk of Holstein and Tropical Milking Criollo. Agrociencia (Montecillo). 2010;44(5):531-539.

41. Berry SD, Lopez-Villalobos N, Beattie EM, Davis SR, Adams LF, Thomas NL, Ankersmit-Udy AE, Stanfield AM, Lehnert K, Ward HE, Arias JA, Spelman RJ, Snell RG. Mapping a quantitative trait locus for the concentration of $\beta$-lactoglobulin in milk, and the effect of $\beta$-lactoglobulin genetic variants on the composition of milk from Holstein-Friesian $x$ Jersey crossbred cows. NZ Vet J. 2010;58(1):1-5.

42. Losi G, Castagnetti GB, Morini D. Le varianti genetiche della caseina $\mathrm{Ke}$ attitudine del latte alla coagulazione presamica. Latte. 1979;4(4):1062-1068.

43. Morini D, Castagnetti GB, Chiavari C, Grazia L, Losi G, Davoli R, Bosi P. Caseification tests carried out with milk characterized by variants $A$ and $B$ of $\beta$-lactoglobulin in the production of Parmigiano-Reggiano cheese. Sci Tecn Latt-Cas. 1982;33:475-492.

44. Malacarne M, Summer A, Fossa E, Formaggioni P, Franceschi P, Pecorari M, Mariani P. Composition, coagulation properties and Parmigiano-Reggiano cheese yield of Italian Brown and Italian Friesian herd milks. J Dairy Res. 2006;73(2):171-177.

45. De Marchi M, Bittante G, Dal Zotto R, Dalvit C, Cassandro M. Effect of Holstein Friesian and Brown Swiss breeds on quality of milk and cheese. $J$ Dairy Sci. 2008;91(10):4092-4102. 